\title{
OS DIAGNÓSTICOS DE ENFERMAGEM NO ENSINO E NA PESQUISA *
}

\section{Diná de Almeida Lopes Monteiro da Cruz **}

CRUZ, D. de A. L. M. da Os diagnósticos de enfermagem no ensino e na pesquisa. Rev. Esc. Enf. USP, v. 26, n. 3, p. - - , dez., 1992.

Trata-se de aspectos de atualização referentes aos possiveis beneficios de utilização dos diagnosticos de enfermagem propostos pela North American Nursing Diagnosis Association (NANDA) no ensino e de aspectos de atualização sobre as pesquisas em diagnósticos de enfermagem.

UNITERMOS: Diagnósticos de enfermagem; ensino; pesquisa.

\section{Introduçáo}

Os diagnósticos de enfermagem, tal como são propostos pela North American Nursing Diagnosis Association (NANDA 1980), causam a primeira vista, a idéia de algo novo e complicado. Eles têm novidades e complicações, mas também trazem em si aspectos tradicionais da prática de enfermagem e tếm o potencial de serem facilitadores dessa prática.

O processo de enfermagem, visto como uma estrutura para organizar as etapas necessárias para o cuidado de enfermagem, inclui, de forma distinta ou não, um passo que envolve a identificação de situaçōes nas quais se intervém. Apesar de poder receber outras denominações o fato de, nesse passo, se pretender discriminar as situações que necessitam de intervenções de enfermagem, ou que estão fora do âmbito da enfermagem caracteriza, de alguma forma, uma etapa de diagnóstico. Isso é tradicional na enfermagem.

A novidade de que eles se revestem decorre da proposta de uniformização dos termos que designam as situaçōes em que se pretende intervir. Se tradicionalmente temos trabalhado com os sinais e sintomas como diagnósticos de problemas ou necessidades dos pacientes como a culminância da fase que poderíamos chamar de diagnóstica, a terminologia proposta pela NANDA vai além. Esses sinais e sintomas passam a ser indicadores de diagnósticos, de onde decorre a necessidade de explicitarmos as relaçōes existentes entre os diferentes sinais e a pertinência entre eles através da denominação do diagnóstico, o que é inovador quanto ao que temos feito até então.

Uma das complicaçōes que advêm dessa padronização é que a denominação de cada diagnóstico envolve um conceito que deve apreender e comunicar, de

- Apresentado na IV Semana Wanda de Aguiar Horta. São Paulo, 1992.

** Enfermeira. Assistente do Departamento de Enfermagem Médico-Cirúrgica da EEUSP. 
forma sucinta, cada situação que eles pretendem designar. Nesse aspecto as dificuldades são muitas visto que, aos termos propostos podem ser atribuídos diferentes significados. Dessa situação decorre a necessidade de ajustarmos aos termos os significados que sejam, o melhor possível, representativos das situações reais. $O$ atendimento a essa necessidade não é simples pois supera as questões semânticas envolvidas.

Outras dificuldades de uma proposta de padronização decorrem da exigência, inerente a ela, de analisarmos cada diagnóstico e de nos posicionarmos se as situações expressas por elas são situações do âmbito da enfermagem, tanto do ponto de vista real quanto ao ponto de vista normativo. Isto é, cada categoria diagnostica deveria ser analisada quanto a ser uma situação que, na prática, tem sido foco de nossas ações, avaliando se é uma situação que também deveria ser foco de nossas ações, ou que não tem sido e nem deveria ser foco de nossas ações. Assim, cada diagnóstico proposto pela NANDA poderia ter o caráter de ser foco, de dever ser foco ou de não ser e nem dever ser foco de nossas ações. A identificação de situações que não são atendidas por nenhum dos diagnósticos propostos é também uma necessidade que se faz presente e tem sido estimulada desde $o$ início do movimento dos diagnósticos de enfermagem.

As facilidades que os diagnósticos podem proporcionar decorrem também da padronização de termos. Uma vez que haja acordo que um determinado diagnóstico expressa uma determinada situação do paciente, toda vez que ele for citado essa situação será mentalmente delineada favorecendo a comunicação intraprofissional.

Ao nos colocarmos diante de cada diagnóstico sempre surge a questão de como intervir nele, se temos conhecimentos, habilidades e experiência tanto para identificá-lo quanto para propor, realizar e avaliar cuidados a ele referentes. Enquanto não temos palavras que explicitem quais as situações em que pretendemos intervir, não nos sentimos tão pressionados para desenvolver os conhecimentos, habilidades e experiência relacionados a essas situações. CASTILHO (1991) no seu estudo sobre a vivência das enfermeiras na formulação de condutas, comenta que um ponto comum nos relatos foi a necessidade que essas enfermeiras sentem de "buscarem novos conhecimentos para a proposição de ações autónomas e comprovadamente eficazes na resolução dos problemas dos pacientes...". Certamente essa necessidade é compartilhada pela maioria das enfermeiras, mas se não temos uma terminologia que expresse o julgamento que fazemos acerca dos problemas dos pacientes, que expresse as situações nas quais queremos intervir, a busca e organização dos conhecimentos para a proposição de ações fica prejudicada.

Tratar dos diagnósticos de enfermagem no âmbito do ensino e da pesquisa envolve esse clima de ambigüidade entre o novo e o tradicional e entre a complicação e facilitação.

\section{Diagnósticos de Enfermagem no Ensino}

Quanto ao ensino dos diagnósticos de enfermagem no Brasil, até o início de 1992 tínhamos conhecimento apenas do estudo de MARIA (1990) referente ao preparo de enfermeiras para utilização dos diagnosticos de enfermagem que en- 
volveu a realização de um curso sobre o assunto e desencadeou a realização de outros ao nível de educação continuada, e da realização de um curso extracurricular na Escola de Enfermagem da USP em 90(*). No entanto, no II Simpósio Nacional sobre Diagnósticos de Enfermagem realizado em julho de 1992, em João Pessoa, tomamos conhecimento de outras iniciativas de introduzir o assunto no ensino: de MESSIAS (1992) o relato do ensino do processo e diagnóstico de enfermagem em educação continuada na UNICAMP, de NÓBREGA, PEREZ e COLER (1992) sobre o ensino através de cursos de extensão e ainda de NÓBREGA, PEREZ e CUNHA (1992) sobre a implantação dos diagnósticos de enfermagem no ensino das disciplinas de Introdução à Enfermagem e Enfermagem Psiquiátrica do Curso de Graduação da Universidade Federal da Parába. Além dessas, existem outras iniciativas ainda não formalmente divulgadas e outras das quais talvez são saibamos nem informalmente.

Na América do Norte onde organizaram-se os esforços para o desenvolvimento dos diagnósticos de enfermagem, a literatura já trata de aspectos referentes ao seu ensino com várias abordagens. É importante destacar que a American Nurse Association (ANA) assume o diagnóstico e tratamento das respostas humanas a problemas de saúde atuais ou potenciais como atividades centrais da enfermagem (ANA, 1980) e endossa os trabalhos da NANDA (JONES, 1990). Com isso, algumas publicações referentes ao ensino dos diagnósticos de enfermagem tomam como relevante ponto de partida para o empenho nessa área essa determinação da ANA (FREDETTE, O'CONNOR, 1979; WHITLEY, DILLON, 1987). Assim, à despeito de controvérsias existentes sobre os diagnósticos de enfermagem, existe um dispositivo de classe que sustenta que os diagnósticos devam ser utilizados e portanto ensinados.

As publicaçōes tratam da importância do preparo das docentes tanto em termos teóricos quanto de experiência clínica com o uso dos diagnósticos de enfermagem, de estratégias para o ensino, de organização de currículo, da inserção do assunto em nivel de graduação e pós-graduação (FREDETTE, O’NEIL, 1987; NOLAN, 1987; TITLER, 1987; PLUNKETT, OLIVIERI, 1989; SHOEMAKER, 1989; ZERR, 1990).

Acreditando que muitos dos diagnósticos aceitos pela NANDA designam situações clínicas que, na nossa prática, têm sido foco de nossas ações considerarei alguns possíveis benefícios de ensiná-los na graduação.

Em primeiro lugar, os diagnósticos vistos como conceitos descritivos e explanatórios, permitiram aos alunos interpretar os dados da avaliação do paciente. Segundo GORDON (1990), sem tais conceitos cada observação a respeito da situação do paciente pode ficar isolada e não ser relacionada a outras observações. Para a mesma autora esses conceitos permitem que certas observações chamem a atenção como grupos, tal como uma imagem poder ser delineada mentalmente quando se olha uma figura na qual essa imagem não foi intencionalmente ou objetivamente retratada. Tomemos como exemplo a situação em que um aluno em fase inicial de formação, diante de um paciente em pós-operatório imediato identifica que ele tem aumento da freqüência de pulso, diminuição da pressão arterial, diminuição do volume urinário e palidez cutânea, além de outros

(*) "Diagnósticos de Enfermagem: conceito, terminologia, usos e implicações"

Re1: Esc. Enf. USP, 1. 26, n-3, p. 427-34, dcz. 1992 
sinais. Sem ter um conceito que lhe permita ter a sua atenção desperta para o possível significado desses sinais como um grupo de sinais que têm relações entre si, muitas dificuldades sobrevirão para que se consiga aprender a ensinar tais relações. Por outro lado, se ele já tem um conceito que espelhe essas relações, como por exemplo o diagnóstico "diminuição do volume de líquidos", a atribuição de significado tanto aos sinais identificados quanto à relações entre eles ficaria facilitada. Mesmo que ele ainda não tenha aprendido esse conceito a situação de ensino poderia ser favorecida ao utilizá-lo, aumentando a possibilidade de que em outras situações semelhantes, as relações entre esses sinais se manifestem e chamem a sua atenção de forma que esse aluno atribua um significado a elas.

Além de facilitar a interpretação do significado das observações, os diagnósticos também facilitam a comunicação do julgamento numa expressão concisa.

O segundo possível benefício é que os diagnósticos de enfermagem trazem à tona as lacunas de conhecimentos sobre as intervenções de enfermagem. Apesar de, à primeira vista, isso não parecer ser um benefício, o aluno deve ser precocemente posto em contato com essas lacunas, com o caráter ainda embrionário tanto do movimento dos diagnósticos de enfermagem no Brasil, quanto das intervenções de enfermagem.

O terceiro seria de contribuir para o desenvolvimento consistente da formação do enfermeiro. Se, na graduação, cada disciplina abordasse de forma mais aprofundada os diagnósticos prevalentes na clientela a que diz respeito, junto a utilização de conhecimentos e habilidades referentes a diagnósticos já abordados em disciplinas anteriores, os alunos teriam o assunto tratado de forma progressiva e cumulativa o que favoreceria a consistência na sua formação. Esse benefício tem consonância com o pressuposto de NOLAN (1987) de que tendo algum conhecimento e experiência no uso dos diagnósticos de enfermagem desde as etapas iniciais da graduação, explorações mais detalhadas seriam mais facilmente encorajadas tanto na graduação quanto na pós-graduação.

Esses benefícios aqui indicados estão interrelacionados e certamente não esgotam todas as possibilidades bem como não eliminam as dificuldades para alcançá-los a contento.

Sobressaem questões que precisam ser analisadas ao se inserir o ensino dos diagnósticos de enfermagem em qualquer nível da formação do profissional: como nos prepararmos, enquanto docentes, para o ensino do assunto? Quais os possíveis efeitos de introduzí-lo em disciplinas isoladamente? Ainda prevalece a afirmação de FREDETTE, O’NEIL de 1989 de que a pesquisa sobre o processo diagnóstico é insuficiente e que quase nada é encontrado na literatura sobre como desenvolver o ensino clínico dos diagnósticos de enfermagem.

\section{Diagnósticos de Enfermagem na Pesquisa}

A pesquisa sobre diagnósticos de enfermagem também está nos seus estágios iniciais, apesar de já terem decorrido duas décadas da First National Conference on Nursing Diagnoses Classification na América do Norte (McFARLANE, 1991). 
O maior enfoque das pesquisas tem sido na área de identificação e validação dos diagnósticos de enfermagem. Desde o início dos trabalhos da NANDA, incluindo as conferências que precederam a sua organização, os diagnósticos são incluídos na taxonomia por serem considerados suficientemente claros para serem testados e validados. Apesar de se justificar a importância de descobrir novos conceitos (diagnósticos), GORDON (1985) afirma que o desenvolvimento dos conceitos diagnósticos existentes é prioritário. Observando que o refinamento dos diagnósticos é um processo contínuo, ela conclue que tal refinamento deve ser baseado em resultados de pesquisas direcionadas à confiabilidade e validade clínica.

A validação de um diagnóstico consiste em evidenciar que um grupo de características definidoras existe para um diagnóstico em questăo e que esse grupo se repete em diversos pacientes (GORDON, SWEENEY, 1979). Segundo CREASON, (1989), quando se pergunta se um diagnostico é clinicamente válido, queremos saber, em primeiro lugar, se ele está apropriadamente formulado, se exclue outros diagnosticos que podem partilhar algumas das mesmas características definidoras; em segundo lugar, dada uma situação de um paciente em que um diagnóstico é formulado, queremos saber se esse diagnóstico seria também formulado para outros pacientes em situações semelhantes; em terceiro lugar, queremos saber se os dados observáveis foram precisamente representados, isto é, se o diagnóstico representa realmente os comportamentos e características do paciente. $O$ tipo de pesquisa clínica associada a essas questões envolve uma série de problemas conceituais e metodológicos.

Em 1979 GORDON; SWEENEY descreveram três modelos de identificação e validação dos diagnósticos de enfermagem, a saber: o modelo de identificação e validação retrospectiva, o modelo clínico e o modelo de validação pela enfermeira. Em 1986 Fehring apud KIM (1989) propôs modificaçōes nesses modelos descrevendo dois modelos para determinar a validade das denominações dos diagnósticos e um para determinar as correlações etiológicas.

Inicialmente, os métodos utilizados para os estudos de validação foram predominantemente levantamentos de opiniấo de enfermeiras, exames retrospectivos de registros e, em menor extensão, a avaliação prospectiva de pacientes (CHANG, 1989).

Na sua revisão de artigos de pesquisa publicados no período de 1973 a 1987, KIM (1989) verificou que os três modelos propostos por GORDON, SWEENEY (1979) têm sido os mais utilizados nos métodos. Refere também outros métodos para identificar e validar as características definidoras dos diagnósticos que podem servir como guias para outras pesquisas com propósitos semelhantes. Com essa revisão, fundamental para os interessados no assunto, assim como a de GORDON (1985) e KIM (1989) observou que as publicações de pesquisas até meados da década de 80 foram feitas predominantemente nos anais das conferências bienais realizadas pela NANDA, que não são de conhecimento de grande parte das enfermeiras e nem tampouco permitem fácil localização dos seus conteúdos pelos sistemas convencionais de catalogação bibliográfica. Entre as conclusões a que chega com essa revisão destacamos as seguintes:

- que desde a revisão de GORDON (1985) as pesquisas em diagnósticos de en- 
fermagem tiveram um crescimento considerável tanto em quantidade quanto em qualidade;

- que a análise crítica dos estudos de validação revelam grande preocupação dos pesquisadores com questōes de confiabilidade e validade dos instrumentos;

- que o desenvolvimento e validação dos diagnosticos na área de enfermagem em saúde coletiva requerem ainda a organização de esforços dos seus especialistas; $\mathrm{e}$

- que o teste de estruturas conceituais para a taxonomia dos diagnósticos ainda é uma lacuna.

Reflete-se nessa revisão de KIM (1989) a maior tendência em utilizar estratégias relativas a pesquisa quantitativa. As perspectivas para utilização de métodos qualitativos, para o aprimoramento dos quantitativos e para a integração de ambos são apresentadas e discutidas nos anais de uma conferência de métodos de pesquisa em diagnósticos de enfermagem, realizada em 1989 (INVITATIONAL, 1989), assim como nos anais da nona conferência da NANDA, cujo conteudo inclue um capítulo sobre métodos qualitativos (McFARLANE, 1991), um sobre quantitativos (SCHROEDER, 1991), outro sobre métodos integrados (KIM, 1991).

Procurar identificar um perfil das pesquisas sobre diagnósticos de enfermagem realizadas no Brasil ainda não é possível. Se considerarmos as publicações em periodicos nacionais, podemos dizer que ela é numericamente inexpressiva. No entanto, sabemos que em eventos de diversas naturezas têm sido relatados estudos sobre o assunto, mas são de difícil localização para análise. Também têm surgido pesquisas vinculadas à programas de pós graduação, como o de CRUZ (1989) e a de MARIA (1990) em São Paulo, a de NÓBREGA (1991) na Paraíba, a de JESUS (1992) em Ribeirão Preto, além de outras que estão sendo desenvolvidas nessas localidades. Não há como sistematizar a identificação desses estudos e certamente há outros dos quais ainda não temos conhecimento. É fundamental que os pesquisadores de diagnósticos de enfermagem se preocupem em não limitar a divulgação de seus estudos aos relatórios de pesquisas de pós-graduação, a apresentação oral em eventos, ou mesmo aos seus anais, que nem sempre são editados. Embora as limitações sejam grandes por existirem poucos periodicos nacionais de enfermagem que sobrevivem, quando o conseguem, com grandes dificuldades, deve haver um esforço para que esses estudos sejam neles publicados, de forma a facilitar o acesso aos interessados e mesmo a divulgação do assunto. Sem conseguirmos traçar um perfil desses estudos no Brasil que permita analisálos, compromete-se a identificação de lacunas, de posicionamentos divergentes, enfim de todos aspectos que podem contribuir para o desenvolvimento consistente dos diagnósticos de enfermagem no Brasil.

\section{Considerações finais}

Separar um assunto em aspectos de ensino e pesquisa e também de assistência é apenas uma estratégia de localização. Não podemos perder de vista que eles estão interrelacionados, que se influenciam mutuamente. Portanto, todos esses aspectos dos diagnosticos de enfermagem trazem consigo as idéias do novo e do tradicional, do facilitador e do complicador sobre as quais tratamos anteriormente. 
Finalizando, os diagnósticos de enfermagem são uma grande idéia. Uma gande idéia que não resolve e nem pretende resolver todos os problemas da enfermagem, mas que abre possibilidades para o seu desenvolvimento. São apenas possibilidades cuja concretização e alcance só serão conseguidos a partir da disposição e compromisso de dar conteúdo a um assunto com algo de novo, carregado de ambigüidades e que, como tal, envolve árduo trabalho. Apenas contemplá-lo não nos dirá se suas possibilidades podem ou não ser concretizadas.

CRUZ, D. de A. L. M. da Nursing diagnosis in teaching and in research. Rev. Esc. Enf. USP, v. 26, n.3, p. - , dec., 1992 .

This paper refers to possible benefits of nursing diagnosis, as the propposed by North American Nursing Diagnosis Associtaion, in teaching and to issues about research in nursing diagnosis.

UNITERMOS: Nursing diagnosis; teaching; research.

\section{REFERENCIAS BIBLIOGRÁFICAS}

1. AMERICAN NURSE'S ASSOCIATION. Nursing: a social policy statement. Kansas City, 1980.

2. CASTILHO, V. A enfermeira vivenciando a formulação de conduta de enfermagem, 1991. 120p. Disser-tação (Mestrado) - Escola de Enfermagem, Universidade de São Paulo.

3. CHANG, B. L. Reability and construct validity. In: INVITATIONAL CONFERENCE ON RESEARCH METHODS FOR VALIDATING NURSING DIAGNOSIS, 1, Palm Springs, 1989. Proceedings, St. Louis, NANDA/Case Western Reserve University, 1989, p.217-32.

4. CREASON, N.S. Clinical validation of nursing diagnosis. In: INVITATIONAL CONFERENCE ON RESEARCH METHODS FOR VALIDATING NURSING DIAGNOSIS, 1, Palm Springs, 1989. Proceedings, St. Louis, NANDA/Case Western Reserv University, 1989, p. 278-304.

5. CRUZ, D. de A. L. M. da Diagnosticos de enfermagem e padröes funcionais de sauide ahterados de pacientes internados por cardiopatia chagásica crônica, 1989. 92p. Dissertação (Mestrado) - Escola de 'Enfermagem, Universidade de São Paulo.

6. FREDETTE, S.; O'CONNOR, $\mathrm{K}$. Nursing diagnosis in teaching and curriculum planning. Nurs. Clin. North Am, v. 14, n. 3, p.541-52, 1979.

7. FREDETTE, S.; ONEIL, M. S. Can Theory improve diagnosis? an examination of the relationship between didatic content and the ability to diagnose in clinical practice. In: Mclane, A (ed.) Classification on Nursing Diagnoses: proceedings of the Seventh Conference of the North American Nursing Diagnoses. St. Louis, Mosby, 1987, p.423-5.

8. GORDON, M. Nursing diagnosis. Annu. Rev. Nurs. Res., v. 3, p.127-45, 1985.

9. GORDON, M. Toward theory: based diagnostic categories. Nurs. Diagn., v. 1, n. 1, p.5-11, 1990.

10. GORDON, M.: SWEENEY, M. A. Methodological problems and issues in identifying and standardizing nursing diagnosis. $A N D$. Adv. Nurs. Sci., v. 2, n. 1, p.1-15, 1979.

11. INVITATIONAL CONFERENCE ON RESEARCH METHODS FOR VALIDATING NURSING DIAGNOSIS, 1, Palm Springs, 1989. Proceedings, St. Louis, NANDA/Case Western Reserve University, 1989.

12. JESUS, C. A. C. de Assistencia de enfermagem a clientes hematologicos: uma visão sistemica, 1992. 279p. Dissertação (Mestrado) - Fscola de Finfermagem de Ribeirão Preto, Universidade de São Paulo. 
13. JONES, P. E. Future directions. In: INVITATIONAL CONFERENCE ON RESEARCH METHODS FOR VALIDATING NURSING DIAGNOSIS, 1, Palm Springs, 1989. Proceedings, St. Louis, NANDA/Case Western Reserve University, 1989, p.324-8.

14. KIM, M. J. Nursing diagnosis. Annu. Rev. Nurs. Res., v. 7, p.117-43, 1989.

15. KIM, M. J. Integrated methods for nursing diagnostic research. In: CARROL-JOHNSON, R. M. (ed.). Classification of Nursing Diagnosis: proceeding of the Ninth Conference of the North American Nursing Diagnosis Association. Philadelphia, J. B. Lippincott, 1991, p. 201-6.

16. MARIA, V.L.R. Preparo de enfermeiros para utilização de diagnosticos de enfermagem: relato de experiência, 1990. 130p. Dissertação (Mestrado) - Escola de Enfermagem, Universidade de São Paulo.

17. McFARLENE, E. A. Qualitative research methods for nursing diagnostic research. In: CARROLJOHNSON, R. M. (ed.)Classification of nursing Diagnosis: proceedings of the Ninth Conference of the North American Nursing Diagnosis Association. Philadelphia, Linpincott, 1991. p. 185-91.

18. MESSIAS, D. K. H. Ensino do processo e diagnচstico de enfermagem ao nivel de educação continuada/Apresentado ao 2 Simpósio Nacional sobre Diagnósticos de Enfermagem, João Pessoa, 1992.

19. Nóbrega, N. M. L. da Diagnósticos de enfermagem da NANDA e a teoria das necessidades humanas basicas de HORTA. 1991. 100p. Dissertação (Mestrado) - Centro de Ciências da Saúde, Universidade Federal da Paraíba.

20. NÓBREGA, N. M. L. PÉREZ, V. L. A. B.; COLER, M. S. Ensino dos diagnósticos de enfermagem através de cursos de extensão/Apresentado ao 2. Simpósio Nacional sobre Diagnósticos de Enfermagem, João Pessoa, 1992.

21. NÓBREGA, M. M. L. DA: PÉREZ, V. L. A. B.; CUNHA, M. M. L. C. Implantação dos diagnósticos de enfermagem no ensino de graduação em enfermagem/Apresentado ao 20 Simpósio Nacional sobre Diagnósticos de Enfermagem, João Pessoa, 1992/

22. NOLAN, T. Utilizing nursing diagnosis in nursing education. Aust. J. Adv. Nurs., v. 4, n. 2, p.51-7, 1987.

23. PLUNKETT, E. J.; OLIVIERI, R. J. A strategy for introducing reasoning hypothesis testing using a simulation approach. Nurse Educ. v. 14, n. 6, p.27-31, 1989.

24. SHOEMACKER, J. K. Nursing diagnosis in graduate curricula. J. Prof. Nurs., v. 5, n. 3, p.140-3, 1989.

25. SCHROEDER, M. A. Quantitative methods for nursing diagnosis research. In: CARROLJOHNSONS, R. M. (ed.) Classification of nursing Diagnosis: proceedings of the Ninth Conference of the North American Nursing Diagnosis Association. Philadeplhia, J. B. Lippincott, 1991, p.192-200.

26. TITLER, M. G. Implementation of nursing diagnoses in nursing education. Nurs. Clin. North Am, v. 22, n. 4, p.987-99, 1987.

27. WHITTLEY, G.; DILLON, A. An idea whose time has come: nursing diagnosis in nursing education. Chart., v. 84, n. 4, p.3-4, 1987.

28. ZERR, S. J. R. Developing strategies for teaching nursing diagnosis. Can. J. Nurs. Res., v. 22, n. 1, p.61-9, 1990. 